\title{
LOS EMPUJES DEL MATERIAL ALMACENADO EN SILOS
}

\section{(LOADS OF STORED BULK SOLIDS IN SILOS)}

\author{
Francisco Ayuga, Prof. Titular de Universidad \\ ETS de Ingenieros Agrónomos de Madrid
}

Fecha de recepción: $15-$ III-95

$582-16$

ESPAÑA

\section{RESUMEN}

Durante un siglo el cálculo de silos ha sido objeto de investigación científica, técnica y aplicada y se han producido grandes avances. Todo este desarrollo no siempre ha sido bien dado a conocer entre los técnicos. El objeto de este articulo es precisamente presentar las técnicas de cálculo de acciones del material almacenado en silos, presentary comentar la normativa, introducir los últimos trabajos que en el cálculo de silos se están haciendo en todo el mundo basados en la técnica de los elementos finitos y aventurar los cambios que esto va a suponer en un futuro.

\section{SUMMARY}

The design of silos has been the object of scientific and technical research for a whole century and this has led to great advances in the field. The results have not always been adequately disseminated among the technicians. The aim of this paper is to present the techniques for calculation of the loads of the materials stored in silos, inform about and comment on the standards, introduce the latest works carried out worldwide based on the finite elements method and, lastly, venture an opinion on the changes this may cause in the future.

\section{Introducción.}

Los primeros grandes silos se empiezan a construir en el último tercio del siglo XIX, normalmente de tablones de madera, pero alcanzando alturas de hasta $20 \mathrm{~m}$. Inicialmente, su cálculo se hacía considerando la presión horizontal hidrostática del grano, exactamente igual que si se tratara del almacenamiento de un líquido, pero pronto se intuyó que las presiones resultantes eran excesivamente grandes, debido al rozamiento del grano con las paredes. Pronto las capacidades de los silos empezaron a aumentar, y cambiaron los materiales empleados en su ejecución. Comenzó así un interés internacional por este tipo de estructuras basado en tres razones principales y alguna más secundaria. Estas razones son:

-Interés económico. El almacenamiento de productos a granel es fundamental en el mundo industrial y se realizan grandes inversiones públicas y privadas en estructuras de almacenamiento en todos los países del mundo.

-Interés científico. Los silos son estructuras complejas donde se combinan comportamientos estructurales de distintos materiales y con muy diversa situación, por lo que todavía hoy después de un siglo de investigación existen grandes lagunas de conocimiento que animan a numerosos investigadores de todo el mundo. Como suele suceder en estos casos, cuanto más se avanza, más lejano se ve el final.

-Interés social. Durante muchos años los silos han sido protagonistas de un gran número de fallos, hundimientos, explosiones, agrietamientos etc., debido al mal conocimiento de su comportamiento estructural. Seguramente es el tipo de estructura con más porcentaje de siniestralidad.

Las distintas aportaciones en investigación y tecnología que de todos los países se iban realizando, generalmente llegaban al común conocimiento de los técnicos a través de las distintas normas que para el cálculo de silos se han ido elaborando. En este sentido es de lamentar que España sea de los pocos países que no dispone de normativa propia, salvo una referencia trivial en la norma MV-101-1962, ahora denominada NBE-AE 88. En la actualidad está ya aprobado el primer borrador del apartado 4 (acciones en silos y depósitos) del Eurocódigo 1 (acciones y bases de diseño en las estructuras)[1], que prontō será norma europea y por tanto española. 
Dentro del capítulo de normas internacionales deben destacarse las alemanas [2], americanas [3 y 4], francesas [5 y 6], canadienses o rusas. Todas ellas resumen y reflejan el bagaje de conocimientos adquiridos, pero sólo de aquéllos ya asentados, aceptados por la comunidad científica internacional y contrastados en obras reales. Los últimos avances quedan siempre a la esperay tardan algún tiempo en ser incorporados a las normas.

La primera aportación importante se produjo en el año 1895 con la publicación de Janssen [7] en la que por primera vez se expone la teoría del "efecto silo", es decir, el material granular roza con las paredes del silo, transmitiéndole parte de su peso por el rozamiento, disminuyendo la presión vertical, y por tanto, también los empujes. Esta publicación cambió en pocos años el concepto del cálculo de silos, que nunca más se realizó como si de un líquido se tratara.

Esta teoría sirvió de base para las primeras normas de cálculo y las posteriores investigaciones que se realizaron. Algunos años después fue criticada ante los fallos que se produjeron en muchos silos y se comenzó a sospechar que la situación de vaciado de silos, con una gran cantidad de material puesto en movimiento, modificaba notablemente los empujes del grano. En la década de los cuarenta los hermanos Reimbert [8] realizaron importantes aportaciones en este sentido y elaboraron una nueva teoría de cálculo que se hizo muy popular. En los años posteriores se investigaron profusamente las situaciones de vaciado y el comportamiento estructural del silo, manteniéndose vivas las teorías de Janssen y Reimbert con algunas adaptaciones y abandonándose progresivamente las demás. En el tema del vaciado fueron muy importantes las aportaciones de Jenike[ 9].

En estos años se iniciaron también las investigaciones con medida experimental de presiones en silos, unas sobre silo real y otras sobre maquetas en tamaño reducido que, en algunos casos, confirmaron los resultados teóricos, pero que en el tema del vaciado oscurecieron aún más el problema, al producirse resultados contradictorios. En este campo destacó el recientemente fallecido investigador español Juan Ravenet.

En la década de los setenta las aportaciones más novedosas se realizaron en el estudio teórico de los detalles estructurales. En Canadá se comenzó al fínal de la década a trabajar en el cálculo de silos mediante el método de los elementos finitos por parte del ingeniero Jofriet y su equipo.

En la década de los ochenta se revisaron prácticamente todas las normas internacionales, incorporando los últimos avances técnicos. Se apreció una vuelta hacia la teoría estática de Janssen, generalizada y complementada con los trabajos posteriores. El método de los elementos finitos se convirtió en la línea de investigación más importante en esta década, junto con el estudio de las propiedades de los materiales en laboratorio, en especial aquéllas necesarias para el cálculo teórico cuya determinación no es convencional.

En la actualidad se han diversificado los trabajos de investigación, pero coexisten tres grupos claramente diferenciados: los que estudian las acciones y deformaciones en silos reales o en maquetas, los que se dedican a mejorar los modelos de comportamiento de los materiales y los que avanzan en el estudio de los esfuerzos estructurales, tanto estáticos como dinámicos y en el estudio de los detalles, normalmente basándose en el método de los elementos finitos (m.e.f.).

\section{El método clásico de cálculo. La teoría de Janssen.}

Las hipótesis en que se basa esta teoría son:

- Las presiones horizontales son constantes en superficies horizontales.

- El valor de $\delta$ (ángulo de rozamiento material-pared) es constante.

- El peso específico del material es uniforme.

- Las paredes del silo son completamente rígidas.

- La relación entre presiones horizontales y verticales es constante para cada altura de silo $\mathrm{k}=\mathrm{p}_{\mathrm{h}} / \mathrm{p}_{\mathrm{v}}$

- Se puede aplicar las teoría de Mohr-Coulomb y Rankine para materiales granulares. Posteriormente diversos investigadores establecieron que, en este caso, el coeficiente $\mathrm{k}$ variará en función de la rugosidad de las paredes entre:

$$
\mathrm{k}=\frac{1-\operatorname{sen} \varphi}{1+\operatorname{sen} \varphi}
$$

para el caso de paredes absolutamente lisas en las que $\delta=0$ y

$$
\mathrm{k}=\frac{1-\operatorname{sen}^{2} \varphi}{1+\operatorname{sen}^{2} \varphi}
$$

para paredes muy rugosas en las que $\delta=\varphi$

En los casos intermedios

$$
\begin{aligned}
& \mathrm{k}=\frac{1-\mathrm{m} \cdot \operatorname{sen} \varphi}{1+\mathrm{m} \cdot \operatorname{sen} \varphi} \cdot \cos ^{2} \delta \\
& \mathrm{m}=\sqrt{1-\mu^{2}} \\
& \mu=\frac{\operatorname{tg} \delta}{\operatorname{tg} \varphi}
\end{aligned}
$$

Ninguna de estas hipótesis es cierta, pero el resultado de hacerlas es suficientemente correcto para silos altos en estado estático y de materiales poco compresibles, como los granos. 
El equilibrio del elemento de altura dz del material de peso específico aparente $\gamma$ queda establecido entre las presiones verticales $p_{v}$ y $p_{v}+d p_{v}$ y las debidas al rozamiento producido por el empuje $\mathrm{p}_{\mathrm{h}}$ sobre las paredes. (Figura 1)

Si A es el área de la sección isobara del silo, U el perímetro $y \operatorname{tg} \delta$ el coeficiente de rozamiento, queda:

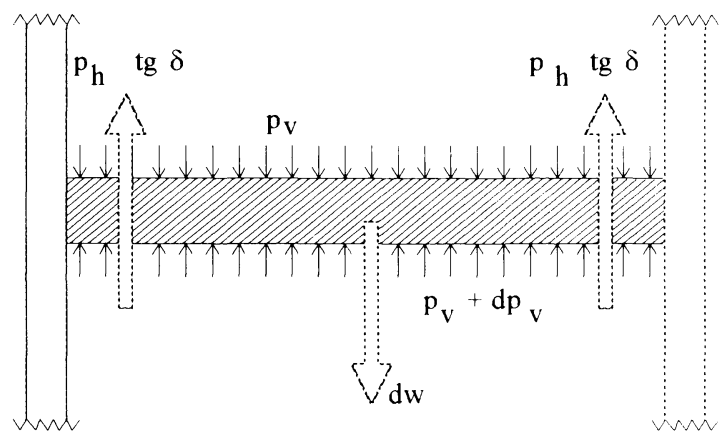

Figura 1

$p_{h} \cdot \operatorname{tg} \delta \cdot d z \cdot U+\left(p_{v}+d p_{v}-p_{v}\right) \cdot A-\gamma \cdot A \cdot d z=0$

$k \cdot d p_{v}=d p_{h} \quad$ y separando variables:

$\left[\frac{A}{k} \cdot d p_{h}=\left(\gamma \cdot A-p_{h} \cdot \operatorname{tg} \delta \cdot U\right) \cdot d z\right]$

$\frac{\mathrm{A}}{\mathrm{U}} \cdot \frac{1}{\mathrm{k}} \cdot \frac{1}{\operatorname{tg} \delta} \cdot \frac{\mathrm{dp}_{\mathrm{h}}}{\frac{\gamma}{\operatorname{tg} \delta} \cdot \frac{\mathrm{A}}{\mathrm{U}}-\mathrm{p}_{\mathrm{h}}}=\mathrm{dz}$

integrando:

$\frac{-\mathrm{A}}{\mathrm{U}} \cdot \frac{1}{\mathrm{k}} \cdot \frac{1}{\operatorname{tg} \delta} \cdot \mathrm{L}\left(\frac{\gamma}{\operatorname{tg} \delta} \cdot \frac{\mathrm{A}}{\mathrm{U}}-\mathrm{p}_{\mathrm{h}}\right)=\mathrm{z}-\mathrm{Cte}$

Sabiendo que para $\mathrm{z}=0 \quad \mathrm{p}_{\mathrm{h}}=0$

$\mathrm{Cte}=\frac{\mathrm{A}}{\mathrm{U}} \cdot \frac{1}{\mathrm{k}} \cdot \frac{1}{\operatorname{tg} \delta} \cdot \mathrm{L}\left(\frac{\gamma}{\operatorname{tg} \delta} \cdot \frac{\mathrm{A}}{\mathrm{U}}\right)$;

$\frac{\mathrm{A}}{\mathrm{U}} \cdot \frac{1}{\mathrm{k}} \cdot \frac{1}{\operatorname{tg} \delta} \cdot \mathrm{L}\left(\frac{\gamma}{\operatorname{tg} \delta} \cdot \frac{\mathrm{A}}{\mathrm{U}}-\mathrm{p}_{\mathrm{h}}\right)=$

$=\frac{\mathrm{A}}{\mathrm{U}} \cdot \frac{1}{\mathrm{k}} \cdot \frac{1}{\operatorname{tg} \delta} \cdot \mathrm{L}\left(\frac{\gamma}{\operatorname{tg} \delta} \cdot \frac{\mathrm{A}}{\mathrm{U}}\right)-\mathrm{z}$

en donde:

$$
1-\frac{\mathrm{p}_{\mathrm{h}}}{\frac{\gamma}{\operatorname{tg} \delta} \cdot \frac{\mathrm{A}}{\mathrm{U}}}=\mathrm{e}^{-(\mathrm{U} / \mathrm{A} \cdot \mathrm{k} \cdot \mathrm{tg} \delta) \mathrm{z}}
$$

resulta finalmente:

$\mathbf{p}_{\mathrm{h}}=\frac{\gamma}{\operatorname{tg} \delta} \cdot \frac{\mathrm{A}}{\mathrm{U}} \cdot\left(1-\mathrm{e}^{-(\mathrm{U} / \mathrm{A} \cdot \mathrm{k} \cdot \operatorname{tg} \delta) z}\right)$

(c) Consejo Superior de Investigaciones Científicas Licencia Creative Commons 3.0 España (by-nc)
Esta curva exponencial tiene un valor asintótico cuando $\mathrm{z} \rightarrow \infty$, que además representa el valor máximo de $\mathrm{p}_{\mathrm{h}}$.

$$
\mathrm{p}_{\max }=\frac{\gamma \cdot \mathrm{r}}{\operatorname{tg} \delta}
$$

donde $\mathrm{r}$ es el radio hidráulico de la sección del silo.

$$
r=\frac{A}{U} \quad p_{\max }=\frac{\gamma \cdot r}{\operatorname{tg} \delta}
$$

(ver figura 2)

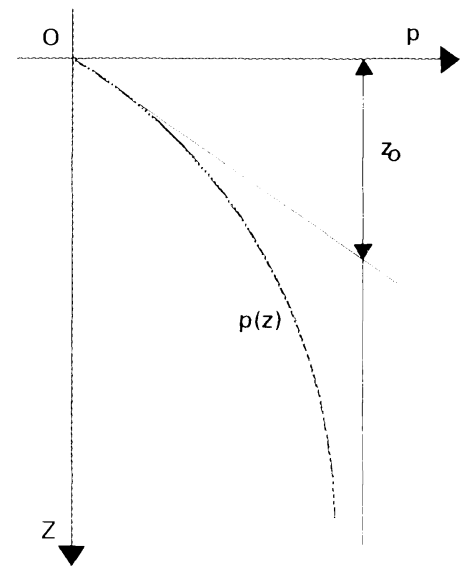

Figura 2

Si $\mathrm{z}_{\mathrm{o}}$ es la ordenada característica, o punto de corte de la tangente en el origen y la asíntota, sabemos:

$$
\begin{gathered}
\mathrm{p}_{\max }=\mathrm{z}_{0} \cdot\left(\mathrm{dp}_{\mathrm{h}} / \mathrm{dz}\right)(\mathrm{z}=0) \\
\frac{\gamma \cdot \mathrm{r}}{\operatorname{tg} \delta}=\mathrm{z}_{0} \cdot \gamma \cdot \mathrm{k}
\end{gathered}
$$

en donde:

$$
\mathrm{z}_{0}=\frac{\mathrm{r}}{\mathrm{k} \cdot \operatorname{tg} \delta}
$$

y queda finalmente:

$$
p_{h}=p_{\max } \cdot\left(1-e^{-2 / z}\right)
$$

Naturalmente:

$$
\mathrm{p}_{\mathrm{v}}=\frac{\mathrm{p}_{\mathrm{h}}}{\mathrm{k}}=
$$


Esta teoría fue extensamente discutida y aplicada y, con algunas modificaciones, constituye labase de la mayoría de las normas de cálculo de silos existentes. En los años cuarenta fue muy criticada al estudiarse y demostrarse que durante el vaciado de los silos se producían presiones horizontales mucho mayores que las obtenidas mediante esta teoría. Se llegaron a medir valores de 2,25 veces los obtenidos por la curva de Janssen y esto hizo proliferar otros métodos de cálculo, entre los que llegó a ser muy popular el de los hermanos Reimbert. Sin embargo, ningún método explicaba adecuadamente el fenómeno del vaciado, por lo que finalmente la ecuación de Janssen sigue siendo básica.

\section{El problema del vaciado en los silos.}

En la mayoría de los sistemas de almacenamiento a granel constituye un problema establecer un modo racional de extraer los productos para su transporte o consumo. Como ya se ha explicado, además de las dificultades del diseño de equipos adecuados y de la gran variedad de ellos que existe para los distintos materiales, se generan sobre la estructura del silo esfuerzos adicionales por el vaciado cuya ley está aún hoy día por esclarecer, pese al gran número de teorías, datos e investigaciones en esa dirección.

Sin embargo, parece que, poco a poco, se van clarificando algunas cosas. Por ejemplo, parece ya universalmente admitido que la aparición de sobreesfuerzos y su magnitud está relacionada con los tipos de flujo de vaciado que se producen. Esta relación fue puesta de manifiesto ya por Jenike en 1960 [9]. Los tipos de flujo son:

-Flujo másico. Toda la masa ensilada se pone en movimiento simultáneamente; alrededor de la salida aumenta la velocidad del material. Las sobrepresiones en las paredes son grandes.

-Flujo de canal. Se forma un canal o cono en el centro de la masa que se desplaza hacia la salida, mientras el resto del material permanece inmóvil junto a la pared. Este tipo de flujo produce sobrepresiones menores, pero es muy peligroso porque se pueden formar bóvedas junto a la tolva, que interrumpen el flujo de vaciado y que, al caer, pueden aumentar enormemente los esfuerzos verticales y horizontales (con un efecto "pistón" si la boca de salida está cerrada).

-Flujo interno. Es un tipo de flujo de canal en el que el canal interno no corta a la pared del silo, sino que asciende hasta la superficie. Es propio de materiales cohesivos como las harinas o las cenizas. (Figura 3)

La obtención de uno u otro tipo de flujo depende de la inclinación máxima de la tolva de vaciado y del ángulo de rozamiento del material con las paredes de la tolva. Se suelen usar gráficos como el de la figura 4 (tomado del Eurocódigo 1 europeo) para su determinación.

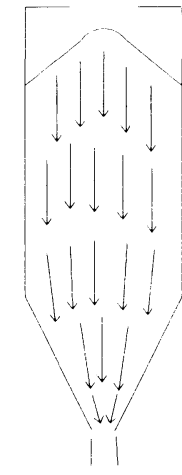

flujo másico

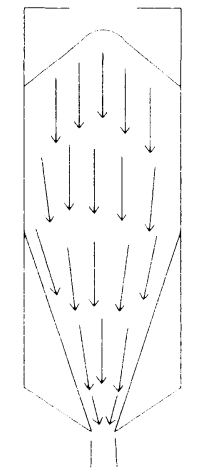

flujo de canal

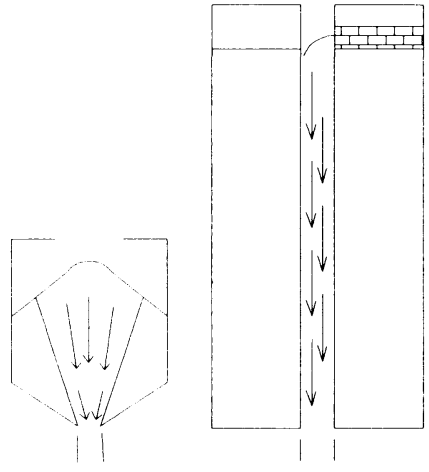

flujo interno
Figura 3

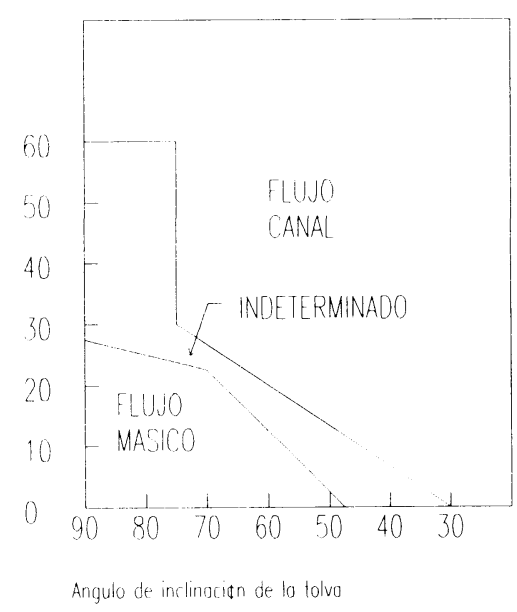

Figura 4

También parece cada vez más evidente que la aparición de sobrepresiones de vaciado está asociada a un cambio de estado del material, similar al paso de un estado activo de presiones de Rankine a un estado pasivo. Este cambio no sería instantáneo y uniforme, sino que se realizaría de forma progresiva, dando lugar a una fuerza de equilibrio, que actuaría en forma de carga localizada y que se propagaría por la pared como una onda.

A todo esto se une que, con frecuencia, el vaciado no se produce por el centro de la sección. La excentricidad del vaciado o el vaciado por bocas múltiples dificultan aún más el estudio del comportamiento del material en el vaciado.

Todo este tema está aún bajo investigación $y$, por el momento, las normas sobre silos resuelven el problema mediante la aplicación de coeficientes de sobrepresión por vaciado, que se aplican para el cálculo de la presión horizontal y, en algunos casos, mediante la aplicación de presiones locales de posición indeterminada y que dependen de la excentricidad del vaciado, el tipo de flujo y otros factores. 
Los coeficientes de sobrepresión dependen, en las normas europeas, del material ensilado y, en las americanas, de las dimensiones del silo y la profundidad estudiada.

\section{Las normas de cálculo de silos.}

Las normas de cálculo de silos tratan de recoger los conocimientos adquiridos a lo largo de años de estudio y de exponerlas, de forma sencilla, para su aplicación práctica. Sorprendentemente en España no existe normativa oficial ni sectorial sobre cálculo de silos, a pesar de ser uno de los países con mayor cantidad y tamaño de silos. Por esto la norma que más nos interesa a los españoles es la recientemente aprobada norma europea [1], que constituye el apartado 4 del Eurocódigo 1 sobre bases de diseño y acciones en estructuras y que, a falta de un posterior desarrollo en nuestro país, será de aplicación en nuestros proyectos. Como su nombre indica, se refiere la norma a la determinación de acciones, por lo que no se dan indicaciones de cálculo estructural ni de detalles constructivos.

Esta norma utiliza para las cargas de llenado de silos el método de Janssen expuesto, aunque sin obtener directamente la relación entre presiones horizontales y verticales, $\mathrm{k}$, con los ángulos de rozamiento interno y de rozamiento grano-pared. Se propugna en la norma la determinación directa de esta constante mediante ensayo del material.

$$
\begin{aligned}
& \mathrm{p}_{\mathrm{wf}}(\mathrm{z})=\gamma \frac{\mathrm{A}}{\mathrm{U}} \mathrm{C}_{\mathrm{z}}(\mathrm{z}) \\
& \mathrm{p}_{\mathrm{hf}}(\mathrm{z})=\frac{\gamma \cdot \mathrm{A}}{\mu \cdot \mathrm{U}} \mathrm{C}_{\mathrm{z}}(\mathrm{z}) \\
& \mathrm{p}_{\mathrm{v}}(\mathrm{z})=\frac{\gamma \cdot \mathrm{A}}{\mathrm{K}_{\mathrm{s}} \cdot \mu \cdot \mathrm{U}} \mathrm{C}_{\mathrm{z}}(\mathrm{z})
\end{aligned}
$$

con los parámetros :

$$
\begin{aligned}
& C_{z}(z)=1-e^{\left(-/ / z_{0}\right)} \\
& z_{0}=\frac{A}{K_{s} \cdot \mu \cdot U}
\end{aligned}
$$

donde :

z profundidad considerada del silo

A sección del silo

$\mathrm{U}$ perímetrodel silo

$\gamma$ peso específico del material almacenado

$\mu$ coeficiente de rozamiento del material y la pared

$\mathrm{K}_{\mathrm{s}}$ relación entre presiones horizontales y verticales

$\mathrm{p}_{\mathrm{wf}}$ presión de rozamiento con la pared durante el llenado

$\mathrm{p}_{\mathrm{hf}}$ presión horizontal durante el llenado

$\mathrm{p}_{\mathrm{v}}$ presión vertical
Para el cálculo de la pared vertical se añade a la presión horizontal de Janssen una carga local actuando en cualquier parte del silo y que es función de la excentricidad relativa de la boca de llenado.

$$
\begin{aligned}
& p_{p}=0,2 \cdot \beta \cdot p_{h f} \\
& \beta=1+4 \cdot \frac{e_{i}}{d_{c}}
\end{aligned}
$$

donde:

e excentricidad de la boca de llenado

$d_{c}$ diámetro del circulo inscrito en la sección del silo

$\mathrm{p}_{\mathrm{p}}$ presión local

La carga se aplica en secciones cuadradas opuestas de dimensión de lado $0,2 . d_{c}$, salvo para silos circulares de pared delgada, en los que se considera la carga en todo el perímetro del silo y una altura $0,2 . d_{c}$, haciendo variar la presión de la forma $p_{p s}=p_{p} \cdot \cos \theta$.

Esta carga puede sustituirse por un valor simplificado. Para el cálculo de la carga de llenado en las tolvas se usa una presión deducida de las siguientes ecuaciones:

$$
\begin{gathered}
p_{n}=p_{n 3}+p_{n 2}+\left(p_{n 1}-p_{n 2}\right) \frac{x}{l_{h}} \\
p_{n 1}=p_{v 0}\left(C_{b} \cos ^{2} \alpha+1,5 \operatorname{sen}^{2} \alpha\right) \\
p_{n 2}=C_{b} \cdot p_{v 0} \cdot \cos ^{2} \alpha \\
p_{n 3}=3 \cdot \frac{A}{U} \cdot \frac{\gamma \cdot K_{s}}{\sqrt{\mu}} \cdot \operatorname{sen}^{2} \alpha
\end{gathered}
$$

donde:

$\mathrm{x}$ distancia desde el punto de salida de la tolva hasta una distancia genérica.

$1_{h}$ longitud total de la pared de la tolva.

$\mathrm{C}_{\mathrm{b}}$ coeficiente de mayoración de la presión vertical $(1,2)$

$\alpha$ ángulo de inclinación de la tolva.

$\mathrm{p}_{\mathrm{v} 0}$ presión vertical en el fondo del silo.

Para el cálculo de los silos en la situación de vaciado la norma europea propone usar los valores de llenado de la siguiente manera:

$$
\begin{aligned}
& p_{w e}=C_{w} \cdot p_{w f} \\
& p_{h e}=C_{h} \cdot p_{h f}
\end{aligned}
$$

con :

$\mathrm{C}_{w}$ Coeficiente de mayoración para rozamiento $(1,1)$ 
$\mathrm{C}_{\mathrm{h}}$ Coeficiente de mayoración para presión horizontal (obtenido de una tabla para cada material,por ej: 1,3 para trigo o 1,4 para cemento)

$\mathrm{p}_{\mathrm{we}}$ presión de rozamiento con la pared durante el vaciado.

$\mathrm{p}_{\mathrm{he}}$ presión horizontal durante el vaciado.

Además de esta carga habrá que considerar una carga local debida a la excentricidad del llenado o del vaciado (la que sea mayor), de la misma forma en que se hace para el caso del llenado.

Para el cálculo de las tolvas en el vaciado en el caso de flujo másico debe considerarse, además, una carga fija concentrada que se extiende en una distancia $0,2 . \mathrm{d}_{\mathrm{c}}$ desde la transición silo-tolva y de valor doble de la presión horizontal de llenado en la transición silo-tolva.

La norma se completa con algunas simplificaciones, cálculo de silos horizontales y de homogeneización, cálculo de depósitos, tablas de características de los materiales y coeficientes de simultaneidad de las cargas e indicaciones sobre métodos de ensayo de los materiales.

Otras normas de interés son las normas DIN alemanas [2] que son la base sobre las que se ha elaborado la norma europea. En algún caso resultan más complejas de aplicación. Por ejemplo, es bastante mayor la carga concentrada en la transición silo-tolva y no es uniforme, sino triangular. La presión local también es más compleja de obtener, basándose en coeficientes que dependen no sólo de la excentricidad del vaciado si no también de las dimensiones del silo, de las paredes y del tipo de material.

Las normas francesas para silos de hormigón [5] también parten de la teoría de Janssen, pero aplicando las modificaciones realizadas por Caquot y Kerisel [10] y considerando el vaciado como el caso de empujes pasivos, con un coeficiente $\mathrm{k}$ básico, $\mathrm{k}=\cos ^{2} \delta$, modificado más tarde si el vaciado es excéntrico. Se consideran, además, temas como los momentos de ovalización de silos cilíndricos, además de los propios del cálculo del hormigón. Las presiones en tolvas son similares a las de las normas alemanas.

Las normas americanas de silos de hormigón [3] consideran válidas las ecuaciones de empujes de Janssen y Reimbert, proponen coeficientes de sobrepresión para el vaciado centrado y no especifican nada para el vaciado excéntrico. Para las tolvas se usa una ecuación muy simplificada que da lugar a presiones uniformes

$q=p_{h} \cdot \operatorname{sen}^{2} \alpha+p_{v} \cdot \cos ^{2} \alpha$

\section{El método de los elementos finitos en el cálculo de silos.}

En la década de los setenta se inició el estudio de los silos mediante el método de los elementos finitos. En un principio se trataba de simular, en lo posible, los modelos teóricos clásicos. Los primeros investigadores se encontraron con muchas dificultades. Para empezar, los programas comerciales existentes en aquella época eran escasos, difíciles de obtener, por su precio o su carácter secreto, y poco flexibles a la hora de adaptarse a las necesidades de los investigadores. Esto hizo que todos ellos trabajaran desde el primer momento sobre programas propios, especialmente adaptados a las investigaciones que desarrollaban. Otra dificultad inicial era la gran potencia de cálculo que se necesitaba y por tanto el alto precio de los equipos básicos. Sin embargo, esto no amedrentó a algunos equipos de trabajo que se iniciaron en esta línea, entre los que se debe destacar a Jofriet[11 ].

Los primeros modelos usaban elementos de tipo plano, cuadrangular, con nodos en los vértices $y$, en algunos casos en los puntos intermedios. La pared se simulaba restringiendo las deformaciones de los nodos. El grano tenía un modelo de comportamiento elástico lineal y en este sentido aparecieron dificultades adicionales, por el total desconocimiento de parámetros básicos, como el módulo de elasticidad o el coeficiente de Poisson de los granos y demás materiales almacenados en silos.

A partir de los primeros trabajos se vio claramente que la línea de investigación era muy prometedora, pero que necesitaba un gran desarrollo. Los primeros esfuerzos se realizaron intentando programar el rozamiento entre el grano y la pared, lo que se hizo, inicialmente, mediante modificaciones en las matrices para los nodos de contacto. Al mismo tiempo iban mejorando las herramientas de cálculoy se podía aumentar el número de elementos y hacer variable su tamaño, con lo que mejoraba mucho la discretización y los resultados obtenidos.

La incorporación de mejoras a estos programas fue acompañada siempre de una enorme rapidez en la evolución de los equipos informáticos, que aún no ha terminado. $\mathrm{Mu}$ chos de los avances conseguidos no hubieran sido posibles sin esta evolución tan destacada.

Pronto se inició el estudio y la incorporación a los programas de modelos de comportamiento de los materiales almacenados, diferentes al elástico lineal. En principio se usaron modelos elastoplásticos, como el de Mohr-Coulomb o el de Drucker-Prager [12]. Más adelante se incorporó el modelo propuesto por Lade [13] que se adaptaba mejor al comportamiento de materiales no cohesivos, como es el caso de los granos. En la figura 5 pueden apreciarse las diferencias entre las tres superficies límite de rotura. En los últimos años se han incorporado por algunos investigadores modelos más complejos, como el de Wilde [14]. Otros grupos de trabajo cambiaron hacia modelos viscoelastoplásticos, como el de Kolymbas [15] o el endocrónico, para simular las propiedades de flujo de los materiales almacenados en silos y poder analizar más adecuadamente los procesos dinámicos del silo: llenado, 
vaciado, vibraciones autoinducidas, etc. En todos los casos existe un gran vacío experimental, respecto alos parámetros necesarios para la adecuada definición de estos modelos (como por ejemplo el ángulo de rozamiento interno, la cohesión, el módulode elasticidad, el ángulo de rozamiento con la pared, la viscosidad, etc.), que se resuelve generalmente con la buena intuición de los investigadores. Los últimos años han proliferado los trabajos en este sentido, a remolque de las necesidades crecientes de los estudios teóricos. Naturalmente, al usar elementos de comportamiento no lineal, se hace necesario utilizar iteraciones de Newton-Raphson para la resolución de las ecuaciones en el programa de elementos finitos.

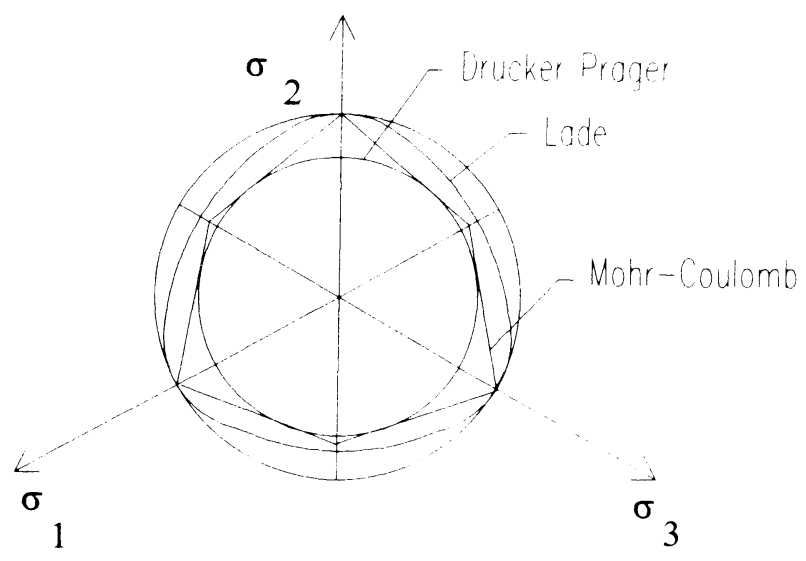

Figura 5

También se ha mejorado en los programas de elementos finitos para el cálculo de silos el contacto entre el material almacenado y la pared del silo. Para ello, en lugar de modificaciones internas del programa, la tendencia moderna es usar elementos especiales de contacto, en los que se tiene en cuenta no solamente el rozamiento, sino también el desplazamiento relativo del grano y la pared.

Algunos investigadores han probado con éxito mallas basadas en elementos diferentes a los cuadrangulares, como por ejemplo los triangulares, y otros han permanecido fieles a los originales. En este sentido existe una notable disparidad de criterios, así como en el tamaño de los elementos ola forma de realizar las discretizaciones. Se ha añadido en algunos programas la simulación del llenado y del vaciado mediante la incorporación o desaparición de capas de elementos de material.

Por último, también se han introducido en los programas las posibles deformaciones de la pared del silo, en especial para el caso de silos cilíndricos de chapa de acero, sobre los que han trabajado la mayoría de los investigadores. Los grandes avances producidos en estos 20 años de trabajo en el comportamiento de diferentes tipos de estructuras y, en este caso concreto, en el comportamiento de los elementos tipo membrana han podido trasladarse a los programas de silos para integrar todo el sistema grano-rozamientopared. Los trabajos de Rotter [16] y Briassoulis [17] han contribuido enormemente al avance en este sentido, permitiendo también el estudio de detalles estructurales, como los elementos de refuerzo contra pandeo y viento, las vigas de transición silo-tolva, etc. Las paredes de hormigón armado han sido mucho menos estudiadas, así como formas de silo distintas de las cilíndricas.

\section{Conclusiones y comentarios.}

-Pese al avance experimentado en el cálculo de las acciones del material almacenado en los silos, aún quedan muchos puntos por aclarar, especialmente respecto al comportamiento en el vaciado.

-Resulta paradójico que España sea de los pocos países avanzados que no dispone de normativa propia para cálculo de silos y más si se tiene en cuenta la cantidad y tamaño de nuestros silos y la calidad de nuestros ingenieros y científicos.

-Las normas de cálculo de silos resuelven de forma práctica los problemas derivados de la aplicación de las teorías clásicas de cálculo, pero no dan indicación alguna respecto a las nuevas técnicas de cálculo por elementos finitos.

-Estas teorías están ya suficientemente avanzadas y sus resultados contrastados, pero necesitan una base importante de características paramétricas de los materiales, que está en estos momentos en investigación.

-Poco a poco se están introduciendo mejoras importantes en la simulación del comportamiento del material ensilado, tanto en situación estática como en movimiento, en el interface material-pared y en el comportamiento de los elementos estructurales.

-Sin embargo, están aún poco estudiados los refuerzos, el pandeo y la abolladura de los silos metálicos cilíndricos y todo lo relacionado con los silos poligonales, baterías de silos, silos de hormigón, silos de piezas prefabricadas, etc. Tampoco se ha abordado aún ,extensamente, la interacción silo-pilar y silo-cimiento.

-Los programas de elementos finitos son específicos para cálculo de silos y necesitan grandes ordenadores para su uso, no obstante, en el estado actual de la técnica, sería factible la utilización de programas comerciales con ordenadores personales. 


\section{BIBLIOGRAFÍA}

[1] ENV 1991-4. Eurocode 1: Basis of design and actions on structures. Part 4 : Actions on silos and tanks.

[2] DIN 1055 part 6 (1987): Design loads for buildings. Loads in silo bins.

[3] ACI313-77(1983): Recommended practice for design and construction of concrete bins, silos, and bunkers for storing granular materials.

[4] ASAE Standar EP-433 (1989): Loads exerted by free flowing grains on bins.

[5] SNBATI (1986): Règles professionnelles de conception et de calcul des silos en béton armé ou précontraint.

[6] P22-630 (1987): Silos métalliques, calcul des actions dans les cellules.

17) Janssen H. A. (1895) "Versuch über Getreidedruck in Sillozellen. Z. VDI. vol 39 pp. 1045-1049.

18] Reimbert, M. (1943) "Recherches nouvelles sur les efforts exercés par les màteries pulverulentes ensilées sur les parois des silos" Annales ITBTP n ${ }^{\circ} 11$ pp. 1-48 discusión pp. $49-60$

[9] Jenike et al. (1960) "Flow properties of bulk solids". ASTM Proc. vol. 60 pp. 1168-1181.

[10] Caquot A. y Kerisel J. (1956) "Traité de Mécanique des
Sols". $3^{\mathrm{a}}$ ed. Gauthier-Villars.

[11] Jofriet J. C. et al. (1977) "Friction model for finite element analyses of silos". Trans. of ASAE . Vol. 20 pp. 735-740.

[12] Drucker, D. C. ; Prager, W. (1952) "Soil mechanics and plastic analysis on limit design" Quart. Appl. Math. vol. 10 n'2 pp. 157-165.

[13] Lade, P.V. (1977) "Elasto-plastic stress strain theory for cohesionless soil with curved yield surfaces". Inter. Jour. of Solids and Structures. Vol. 13 pp. 1019-1035.

[14] Wilde, P. (1979) "Principes mathématiques et physiques des modèles élastoplastiques des materiaux granulaires". Colloque des Ponts et Chausées. París.

[15] Gudheus, G. ; Kolymbas, D. (1979) “ A constitutive law of the Rate Type for soils". Proc. of the 3rd Int. Conf. on Num. Meth. in Geomechanics. Aachen pp. 319-329.

[16] Teng, J. G. ; Rotter, J. M. (1992) "Recent research on the behaviour and design of steel silo hoppers and transition junctions". Jour. of Const. Steel Research vol. 23 pp. 313343.

[17] Briassoulis, D. ; Pecknold, D.A. (1986) "Behaviour of empty steel grain silos under wind loading: part 1, the stiffened cylindrical shell". Eng. Struct. vol. 8 pp. 260-275. 